\title{
Comunidades bentónicas de los fondos aledaños a Jaizkibel (Golfo de Vizcaya)
}

\author{
Jaizkibel inguruko hondoetako komunitate bentikoak (Bizkaiko Golkoa) \\ Benthic communities on bottoms around Jaizkibel (Bay of Biscay)
}

\author{
Florencio Aguirrezabalaga ${ }^{1,2 *}$ \\ ${ }^{1}$ Donostiako Irakasleen U.E., Euskal Herriko Unibertsitatea, UPV-EHU, Oñati plaza 3, 20018 Donostia-S. Sebastián. \\ ${ }^{2}$ S.C. INSUB E.K., Zemoria 12, 3223 P.K., 20013 Donostia-S. Sebastián. \\ *Correspondencia: p.agirrezabalaga@ehu.es
}

\section{RESUMEN}

En este trabajo se sintetiza y resume el conocimiento que se tiene sobre las comunidades bentónicas que habitan los fondos (rocosos y blandos; intermareales y sublitorales) de la zona de Jaizkibel. Para ello, se han revisado los principales trabajos que han sido publicados (artículos, libros) sobre esos temas, así como otros materiales e informes inéditos. Se describen las distintas comunidades bentónicas que han sido identificadas y señaladas en aguas de Jaizkibel, a la vez que se relacionan con las categorías EUNIS (European Nature Information System).

PALABRAS CLAVE: Comunidades bentónicas, EUNIS, fondo blando, fondo rocoso, Golfo de Vizcaya, intermareal, Jaizkibel, sublitora.

\section{LABURPENA}

Lan honetan Jaizkibel inguruko hondoetan (arrokatsuak nahiz bigunak; mareartekoak nahiz mareazpikoak) bizi diren komunitate bentikoei buruz dugun ezagutza laburbiltzen da. Horretarako, gai horien inguruan argitaratu diren lan nabarmenenak (artikuluak, liburuak) eta hainbat argitaratugabeko txosten eta material aztertu eta berrikusi dira. Jaizkibelgo uretan identifikatu eta aipatu izan diren komunitate bentikoak deskribatzen dira eta, era berean, EUNIS (European Nature Information System) kategoriekin erlazionatzen dira.

GAKO-HITZAK: Bizkaiko Golkoa, EUNIS, hondo arrokatsua, hondo biguna, Jaizkibel, Komunitate bentikoak, mareartea, sublitorala

\section{ABSTRACT}

This work summarises the knowledge on the benthic communities inhabiting the bottoms (rocky and soft bottoms; intertidal and sublittoral) situated in the area of Jaizkibel. With this aim, the majority of the publications (papers, books) and unpublished reports and data on this theme have been revised and analized. The different benthic communities identified and designed in Jaizkibel waters are described and, at the same time, they are related with the EUNIS cathegories (European Nature Information System).

KEY WORDS: Bay of Biscay, Benthic communities, EUNIS, intertidal, Jaizkibel, rocky bottom, soft bottom, sublittoral.

\section{INTRODUCCIÓN}

El monte Jaizkibel es una formación montañosa perteneciente a la Cadena Terciaria Costera, que se extiende desde la bocana de Pasajes hasta el Cabo de Higuer. Se sitúa en la parte noreste de Gipuzkoa y da nombre a la Formación Jaizkibel, que es la alineación montañosa que desde Zumaia al Cabo Higuer forma una resistente barrera que separa la costa de las zonas del interior. Formada durante el Eoceno, está constituida por una potente sucesión de gruesos estratos de arenisca cuarzosa, de tonos amarillos y cemento calcáreo, entre los que se intercalan delgados niveles arcillosos o lutitas, de aspecto pizarroso. Esta serie estratigráfica se asienta sobre el denominado Flysch de Gipuzkoa, serie alternante de calizas, areniscas y margas, que se formó durante el Paleoceno. A finales del Eoceno, hace unos 40 millones de años, durante la fase pirenaica de la Orogenia Alpina, estos materiales se pliegan, se elevan del fondo marino y dan lugar a un relieve monoclinal abrupto y escarpado que llega directamente al mar (Torres \& Viera, 1998).

Su vertiente septentrional, enfrentada directamente al mar, presenta un relieve abrupto, con acantilados que en su parte occidental pueden llegar a alcanzar una altura de $240 \mathrm{~m}$. Se va suavizando hacia el este, alternándose con pequeñas calas, donde las acumulaciones detríticas de materiales finos son escasas.

Las características geológicas, geográficas e hidrodinámicas determinan la naturaleza del sustrato donde se desarrollan las comunidades bentónicas, entre las que se distinguen aquellas que habitan fondos blandos (arena, fango...) de las que se desarrollan sobre sustrato rocoso. En Jaizkibel, entre Pasajes y el cabo de Higuer, los fondos marinos predominantes en las aguas más someras son de naturaleza rocosa. Un cinturón rocoso se extiende paralelamente a la línea de costa y perpendicularmente a la dirección predominante de las olas (Galparsoro et al., 2010), llegando hasta la isobata de 20-40 m de profundidad. Apenas algunas zonas arenosas en pequeñas calas o en zonas como Azabaratza, donde se sitúa una zona de acumulación de sedimento (Fontán et al., 2006), rompen la continuidad de estos fondos. A partir de esta profundidad la naturaleza del fondo cambia. En la zona occidental de Jaizkibel, en general, el sustrato está formado por fondos blandos, constituidos por sedimentos de grano fino -arenas, arenas fangosas, fangos arenosos, principal- 
mente,- mientras que en la parte más oriental son más abundantes los fondos mixtos dominados por rocas (Galparsoro et al., 2010).

El hidrodinamismo producido por la acción del oleaje y las mareas es uno de los principales factores que determinan las características de las comunidades macrobentónicas del litoral costero (San Vicente et al., 1992), especialmente de las propias de la zona intermareal. Debido a su orientación N y NO (coincidente con la de los vientos dominantes), a su falta de protección frente al oleaje, y por las características del régimen hidrodinámico prevalente, la costa de Jaizkibel es una costa muy expuesta.

El régimen mareal de la costa vasca es de tipo semidiurno (en un período de tiempo de unas 24 horas se alternan dos mareas bajas con otras dos mareas altas), y tiene una amplitud de marea máxima de 4,4 m en las mareas vivas equinocciales y una mínima de 2,2 $\mathrm{m}$, en las mareas muertas (Iribar \& Ibáñez, 1979).

La costa vasca, situada en la zona más interna del Golfo de Bizkaia, en su ángulo SE, muestra unas características biogeográficas marcadamente distintas a las del resto de las regiones del Golfo, y se caracteriza por la predominancia de especies de aguas cálidas-templadas (de afinidad meridional) y por una menor presencia de especies de aguas más frías (de afinidad septentrional). Estas diferencias quedan reflejadas tanto en la flora como en la fauna, pero son mucho más marcadas cuando se observa la fisionomía de las comunidades algales. La flora que habita los fondos rocosos de la costa vasca se distingue por la ausencia total o casi total (en las zonas intermareal y sublitoral, respectivamente) de las grandes fucáceas y laminariáceas (feofíceas) y por la dominancia de especies de rodofíceas de aguas cálidas-templadas. Así, las densas poblaciones de algas feofíceas, tan abundantes en las costas atlánticas del norte de Europa, desaparecen al llegar a la costa vasca y, progresivamente, vuelven a reaparecer a partir de la zona occidental de la costa de Bizkaia y de la costa de Cantabria, para alcanzar otra vez su mayor desarrollo en las costas gallegas y del norte de Portugal. Fue Sauvageau (1897) quien observó e indicó por primera vez la similitud de la vegetación algal de las costas de Galicia y Bretaña, y su diferencia con la de la costa vasca, que mostraba un carácter más propio de costas más meridionales.

Más adelante, Fischer-Piette (1935, 1938, 1955a, 1955b, 1956) profundizó en el estudio de estas "anomalías" proponiendo una explicación a este fenómeno, que denominó como "meridionalización", al igual que, más tarde, harán Ibáñez (1978, 1989) e Ibáñez et al. (1980). El factor fundamental para explicar estas anomalías biogeográficas es la temperatura (Fischer-Piette, 1935; Borja \& Gorostiaga, 1990). Las diferencias térmicas que se producen entre el interior del Golfo de Bizkaia (costa vasca) y sus extremos (Galicia y Bretaña) son producidas por dos fenómenos distintos. Por una parte, durante la época estival (Abr.-Nov.) se produce un calentamiento de las masas de agua superficiales del interior del Golfo, donde se forma una bolsa de agua más cálida cuya temperatura máxima se alcanza en Ago. Al mismo tiempo, en las costas gallegas se produce un afloramiento de aguas profundas frías y ricas en nutrientes (upwelling) que, comenzando en May., alcanza su máximo en Jul.-Ago. y la primera mitad de Sep., y se extingue en Oct. (Fraga, 1981). Las diferencias térmicas máximas entre las costas vasca y gallega se producen en Ago., y mientras que en la costa de Gipuzkoa la temperatura superficial del agua de mar puede rebasar los $22{ }^{\circ} \mathrm{C}$ (la temperatura media del agua de mar tomada en el Aquarium de Donostia entre 1950 y 1989 fue de 21,9 ํ), en Galicia se sitúa entre 17-19 ㄷ (Servain, 1977; Casares, 1987). Esta conjunción de factores explicaría, por tanto, las diferencias que se observan en las comunidades bentónicas dentro del Golfo de Bizkaia (Díez et al., 2000). Al estar situado Jaizkibel en el punto más interno del Golfo, esta tendencia meridional es muy marcada.

El efecto de todos estos factores condiciona las características de las comunidades bentónicas que habitan los fondos intermareales y submareales. El objetivo de este trabajo es revisar el conocimiento actual sobre las comunidades bentónicas próximas a Jaizkibel.

\section{COMUNIDADES BENTÓNICAS}

Borja et al. (2004), en el capítulo 18 del libro titulado Oceanography and marine environment of the Basque Country (Eds Borja \& Collins), describen las características de la distintas comunidades bentónicas que habitan los fondos marinos de la costa vasca. Excepto las propias de ambientes estuarinos (que las podremos encontrar en el interior del estuario del Bidasoa), en el área de Jaizkibel se desarrollan la mayor parte de las comunidades que se han descrito para los fondos blandos y rocosos de la plataforma continental de la costa vasca. El trabajo citado es una referencia obligada cuando tratamos de comunidades bentónicas de la costa vasca. En el área de Jaizkibel se han llevado a cabo numerosos trabajos con el objeto de estudiar las comunidades bentónicas de sus fondos, entre los que podríamos destacar los realizados por Sarasua et al. (1984), Pérez Celorrio et al. (1985), AZTI-SIO-INSUB-UPV/EHU (1993), Borja et al. (1995) y Martínez et al. (2007).

La descripción de las comunidades se hará distinguiéndolas primeramente en función de las características del sustrato en el que se desarrollan (sustrato blando o rocoso), y, dentro de cada categoría, entre las que habitan la zona intermareal o la zona submareal/sublitoral. En las Tablas 1 y 2 se señala la relación entre las distintas comunidades y los hábitats aceptados por EUNIS.

\section{COMUNIDADES DE FONDO ROCOSO}

\section{Comunidades del intermareal}

Los ecosistemas intermareales se desarrollan en el espacio que queda al descubierto entre la bajamar y la pleamar. La altura y extensión de esta franja intermareal 


\begin{tabular}{|c|c|c|c|c|}
\hline \multicolumn{3}{|c|}{ Clasificación EUNIS } & Descripción & Zonación mareal \\
\hline \multicolumn{5}{|c|}{ B3- Acantilados, cornisas y costas rocosas, incluido el supralitoral } \\
\hline $\begin{array}{l}\text { B3.1 Roca supralitoral } \\
\text { (zona de liquenes o } \\
\text { de salpicaduras). }\end{array}$ & $\begin{array}{l}\text { B3.11 Roca } \\
\text { supralitoral y } \\
\text { de la franja } \\
\text { litoral con } \\
\text { líquenes o } \\
\text { pequeñas } \\
\text { algas verdes. }\end{array}$ & $\begin{array}{l}\text { B3.111 Roca } \\
\text { supralitoral con } \\
\text { líquenes amarillos } \\
\text { o grises. } \\
\text { B3.113 Roca de la } \\
\text { franja litoral con } \\
\text { Verrucaría maura. }\end{array}$ & $\begin{array}{l}\text { Costa rocosa que se extiende en la franja supralitoral } \\
\text { (intermareal superior) y en la zona supralitoral ( }>4,4 \mathrm{~m}) \text {. } \\
\text { La parte superior del supralitoral está cubierto por el liquen } \\
\text { Xanthoria parietina, mientras que la inferior la ocupa el } \\
\text { liquen de color negro Verrucaria maura. En los niveles más } \\
\text { bajos se pueden encontrar ejemplares de los moluscos } \\
\text { Melarhaphe neritoides y Patella rustica, y cinturones } \\
\text { estacionales del alga cloroficea Blidingia minima. }\end{array}$ & Intermareal \\
\hline \multicolumn{5}{|c|}{ A1- Roca litoral y otros sustratos duros } \\
\hline \multirow{3}{*}{$\begin{array}{l}\text { A1.1 Roca litoral } \\
\text { de alta energía. }\end{array}$} & $\begin{array}{l}\text { A1.11 Comuni- } \\
\text { dades de } \\
\text { mejillones y/o } \\
\text { balanos. }\end{array}$ & $\begin{array}{l}\text { A1.112 Chthamalus } \\
\text { spp en rocas del } \\
\text { litoral superior de } \\
\text { zonas expuestas. }\end{array}$ & $\begin{array}{l}\text { Compacto cinturón del cirripedo Chthamalus stellatus que } \\
\text { cubre la zona mediolitoral superior de la costa rocosa. }\end{array}$ & \multirow{4}{*}{ Intermareal } \\
\hline & \multirow{2}{*}{$\begin{array}{l}\text { A1.12 Comuni- } \\
\text { dades de fucá- } \\
\text { ceas robustas } \\
\text { y/o algas rojas. }\end{array}$} & $\begin{array}{l}\text { A1.121 F. spiralis } \\
\text { f.limitaneus en } \\
\text { rocas del litoral } \\
\text { superior de zonas } \\
\text { muy expuestas. }\end{array}$ & $\begin{array}{l}\text { Aunque poco común en la costa vasca, sin embargo en } \\
\text { paredes subverticales muy batidas de la zona mediolitoral } \\
\text { superior el alga parda Fucus spiralis var. limitaneus forma } \\
\text { un cinturón bien desarrollado. }\end{array}$ & \\
\hline & & $\begin{array}{l}\text { A1.122 Corallina } \\
\text { elongata (ahora } \\
\text { Ellisolandia } \\
\text { elongata) en } \\
\text { rocas del litoral } \\
\text { superior de zonas } \\
\text { muy expuestas. }\end{array}$ & $\begin{array}{l}\text { Comunidad ampliamente extendida desde el mediolitoral } \\
\text { inferior de la costa rocosa hasta el margen de la zona } \\
\text { infralitoral. Está dominada por las alga rojas calcáreas. E. } \\
\text { elongada, que da nombre a la comunidad, y Lythophyllum } \\
\text { incrustans, que tapiza las rocas. Otras especies abundan- } \\
\text { tes son las algas Cladostephus spongiosus, Ceramium sp. } \\
\text { y Jania rubens, y las lapas P.vulgata y P. depressa. }\end{array}$ & \\
\hline $\begin{array}{l}\text { A1.4 Estructuras } \\
\text { singulares de } \\
\text { roca litoral. }\end{array}$ & $\begin{array}{l}\text { A1.41 Comuni- } \\
\text { dades de char- } \\
\text { cas litorales. }\end{array}$ & $\begin{array}{l}\text { A1.411 Charcas } \\
\text { litorales domindas } \\
\text { por incrustaciones } \\
\text { de coralina. }\end{array}$ & $\begin{array}{l}\text { Pozas pequeñas y someras del eulitoral en costas expues- } \\
\text { tas y muy expuestas, que están tapizadas por algas } \\
\text { calcáreas, entre las que destacan E. elongata y } \\
\text { L. incrustans. Pueden albergar importantes poblaciones } \\
\text { del erizo Paracentrotus lividus, que en las rocas más } \\
\text { blandas forman cavidades redondeadas donde habitan. }\end{array}$ & \\
\hline \multicolumn{5}{|c|}{ A3- Roca infralitoral y otros sustratos duros } \\
\hline \multirow{3}{*}{$\begin{array}{l}\text { A3.1 Roca infralitoral } \\
\text { de alta energía }\end{array}$} & \multirow{2}{*}{$\begin{array}{l}\text { A3.15 Comuni- } \\
\text { dades algales } \\
\text { frondosas } \\
\text { (que no sean } \\
\text { Laminariáceas). }\end{array}$} & $\begin{array}{l}\text { A3.152 Gelidium } \\
\text { comeum en fondo } \\
\text { rocoso y rocas } \\
\text { infralitorales muy } \\
\text { expuestas. }\end{array}$ & $\begin{array}{l}\text { Se sitúa entre 0-10/15m de profundidad en costas rocosas } \\
\text { expuestas a la acción de las olas con baja o moderada } \\
\text { sedimentación de arena. Puede extenderse hasta los } 25 \mathrm{~m} \\
\text { de profundidad mezclada con el alga parda Cystoseira } \\
\text { baccata. Normalmente son abundantes las algas epifitas } \\
\text { en primavera (Dyctiota dichotoma) y verano (Plocamium } \\
\text { cartilagineum). Las rocas permiten la existencia de otras } \\
\text { especies como Mesophyllum lichenoides, Zanardinia } \\
\text { prototypus, E. elongada, Rhodymenia pseudopalmata } \\
\text { y Cryptopleura ramosa. }\end{array}$ & Sublitoral \\
\hline & & $\begin{array}{l}\text { A3.151 Cystoseira } \\
\text { spp en fondos } \\
\text { rocosos y roca } \\
\text { infralitoral expuesta. }\end{array}$ & $\begin{array}{l}\text { Sobre sustrato rocoso que se extiende desde el limite } \\
\text { inferior de la comunidad de Gelidium hasta } 20-25 m \text { de } \\
\text { profundidad con tasa de sedimentación baja. Normalmente } \\
\text { cubierta por distintas comunidades bentónicas que forman } \\
\text { una zonación continua de macroalgas y macrobentos, limitada } \\
\text { por la profundidad, turbidez, tasa de sedimentación, } \\
\text { orientación del estrato y la exposición. En zonas expuestas } \\
\text { la zonación se describe como Cystoseira baccata, C.baccata } \\
\text { - Laminaria ochroleuca (5-20m) y H.flicina (20-25m). }\end{array}$ & \multirow{4}{*}{ Sublitoral } \\
\hline & $\begin{array}{l}\text { A3.22 Comunida- } \\
\text { des de Laminariá- } \\
\text { ceas y algas en } \\
\text { zonas protegidas } \\
\text { de las corrientes } \\
\text { de marea. }\end{array}$ & $\begin{array}{l}\text { A3.226 Halopteris } \\
\text { flicina con costra } \\
\text { coralina sobre rocas } \\
\text { infralitorales en } \\
\text { medios moderada- } \\
\text { mente expuestos. }\end{array}$ & propuesto según EUNIS 2008 & \\
\hline \multicolumn{4}{|c|}{ A4- Roca circalitoral y otros sustratos duros } & \\
\hline $\begin{array}{l}\text { A4.1 Roca circalitoral } \\
\text { moderadamente } \\
\text { expuesta atlántica y } \\
\text { mediterránea. }\end{array}$ & $\begin{array}{l}\text { A4.22 Arrecifes } \\
\text { de Sabellaria } \\
\text { sobre roca } \\
\text { circalitoral. }\end{array}$ & $\begin{array}{l}\text { A4.22 Sabellaria } \\
\text { spinulosa } \\
\text { encostrada sobre- } \\
\text { roca circalitoral. }\end{array}$ & $\begin{array}{l}\text { Este tipo de habitat se encuentra en sustratos rocosos, } \\
\text { rocas y cantos circalitorales de medios moderadamente } \\
\text { expuestos y con corrientes de marea de intensidad } \\
\text { moderada. Se caracteriza porque el sustrato rocoso } \\
\text { está cubierto por una densa costra formada por el } \\
\text { politiqueto Sabellaria spinulosa. }\end{array}$ & \\
\hline
\end{tabular}

Tabla 1. - Comunidades bentónicas de fondo duro y clasificación EUNIS (European Nature Information System).

1. Taula. Hondo gogorretako komunitate bentikoak eta EUNIS sailkapena (European Nature Information System). 


\begin{tabular}{|c|c|c|c|c|}
\hline \multicolumn{3}{|c|}{ Clasificación EUNIS } & Descripción & Zonación mareal \\
\hline \multicolumn{3}{|l|}{ A2-Sedimento litoral } & & \\
\hline $\begin{array}{l}\text { A2.2 Arena y arena } \\
\text { fangosa litoral. }\end{array}$ & $\begin{array}{l}\text { A2.22-Costas } \\
\text { arenosas } \\
\text { desnudas o } \\
\text { dominadas por } \\
\text { anfipodos. }\end{array}$ & $\begin{array}{l}\text { A2.223-Anfipodos y } \\
\text { Scolelepis spp. en } \\
\text { arenas medias-finas } \\
\text { litorales. }\end{array}$ & $\begin{array}{l}\text { El sedimento está constituido por arenas limpias sin apenas } \\
\text { elementos finos. Se sitúan en la parte baja del intermareal y } \\
\text { continúan en la zona sublitoral en zonas expuestas. Man- } \\
\text { tiene un reducido número de especies entre las que domi- } \\
\text { nan los crustáceos, y algunos poliquetos como Scolelepis } \\
\text { mesnili, Dispio uncinata y Nephtys cirrosa. }\end{array}$ & $\begin{array}{l}\text { Intermareal } \\
\text { Sublitoral }\end{array}$ \\
\hline \multicolumn{5}{|c|}{ A5- Sedimento sublitoral } \\
\hline $\begin{array}{l}\text { A5. } 1 \text { Sedimento } \\
\text { grueso sublitoral. }\end{array}$ & $\begin{array}{l}\text { A5.14- Sedi- } \\
\text { mento grueso } \\
\text { circalitoral. }\end{array}$ & $\begin{array}{l}\text { A5.145 Branchios- } \\
\text { toma lanceolatum } \\
\text { en arena gruesa } \\
\text { circalitoral con } \\
\text { restos de conchas. }\end{array}$ & $\begin{array}{l}\text { Sedimento constituido por arenas limpias de grano grueso o } \\
\text { medio. Se sitúa en profundidades circalitorales sujetas a co- } \\
\text { rrientes de fondo de cierta intensidad que impiden la sedi- } \\
\text { mentación de la fracción más fina. La presencia del } \\
\text { cefalocorrado Branchiostoma lanceolatum es una de las } \\
\text { características de la comunidad que lo habita. }\end{array}$ & \multirow{5}{*}{ Sublitoral } \\
\hline \multirow{3}{*}{$\begin{array}{l}\text { A5.2 Arena } \\
\text { sublitoral }\end{array}$} & $\begin{array}{l}\text { A5.23- Arena } \\
\text { fina infralitoral. }\end{array}$ & $\begin{array}{l}\text { A5.231 Arenas } \\
\text { limpias móviles } \\
\text { infralitorales con } \\
\text { fauna escasa. }\end{array}$ & $\begin{array}{l}\text { Arenas limpias infralitorales, que se sitúan a continuación } \\
\text { de la comunidad de Pontocrates arenarium -Eurydice } \\
\text { pulchra. Se caracteriza por su fauna robusta, principal- } \\
\text { mente crustáceos anfipodos (Bathyporeia) y poliquetos } \\
\text { como Nephtys cirrosa. }\end{array}$ & \\
\hline & $\begin{array}{l}\text { A5.24- Arena } \\
\text { fangosa } \\
\text { infralitoral. }\end{array}$ & $\begin{array}{l}\text { A5.242 Fabulina } \\
\text { fabula y Magelona } \\
\text { mirabilis con bival- } \\
\text { vos venéridos y } \\
\text { anfipodos en arena } \\
\text { fina fangosa com- } \\
\text { pacta infralitoral. } \\
\text { A5.244 Spisula sub- } \\
\text { trucata y Nephtys } \\
\text { hombergii en arenas } \\
\text { fangosas someras. }\end{array}$ & $\begin{array}{l}\text { Fondos arenosos situados en profundidades infralitorales } \\
\text { de la plataforma continental. Caracterizados por una mez- } \\
\text { cla de especies de las comunidades de Tellina y Venus, } \\
\text { que dificulta su diferenciación: Tellina fabula, Chamelea } \\
\text { gallina, Echinocardium cordatum, Nephtys cirrosa, Nucula } \\
\text { sulcata, Bathyporeia elegans. }\end{array}$ & \\
\hline & $\begin{array}{l}\text { A5.25- Arena } \\
\text { fina circalitoral. }\end{array}$ & & $\begin{array}{l}\text { Fondos arenosos situados en la transición entre la plata- } \\
\text { forma continental y el comienzo del talud continental. } \\
\text { Caracterizado por la comunidad de Auchenoplax crinita } \\
\text {-Paradiopatra callopae -Ditrupa arietina. Además de las } \\
\text { especies que dan nombre a la comunidad, otras } \\
\text { especies características son los poliquetos Terebellides } \\
\text { stroemi, Galathowenia oculata, Nothria hispanica, y los } \\
\text { moluscos Thyasira ferruginea y Abra longicallus. }\end{array}$ & \\
\hline A5.3 Fango sublitoral & $\begin{array}{l}\text { A5.35- Arena } \\
\text { fangosa } \\
\text { circalitoral }\end{array}$ & & $\begin{array}{l}\text { Fondos formados por sedimentos muy finos de la plataforma } \\
\text { continental. Caracterizados por la presencia de equinoder- } \\
\text { mos ofiúridos del género Amphiura (A. chiajei y A. filiformis). }\end{array}$ & \\
\hline
\end{tabular}

Tabla 2. - Comunidades bentónicas de fondo blando y clasificación EUNIS (European Nature Information System).

2. Taula. Hondo bigunetako komunitate bentikoak eta EUNIS sailkapena (European Nature Information System).

dependerá, principalmente, de la amplitud de marea, de la pendiente de la costa y de la fuerza del oleaje.

En base a los datos obtenidos por Iribar e Ibáñez (1979), y siguiendo los criterios de Lewis (1964), en el intermareal de la costa vasca, y que por tanto en el área de Jaizkibel, pueden distinguirse tres zonas:

- Franja supralitoral: entre 4,4 m y 3,7 m sobre el punto cero de bajamar, que corresponde a la parte más baja de la zona supralitoral. Únicamente queda sumergida bajo el mar durante las pleamares de mareas de intensidad mediaalta. Por encima de esta franja continúa la zona supralitoral, gran parte de la cual se sitúa por encima de la zona de influencia de las mareas, en el dominio terrestre. La influencia del mar sobre la zona supralitoral se produce como consecuencia de las salpicaduras que se forman por el efecto del oleaje y del viento, por lo que su anchura y extensión está estrechamente relacionada con la exposición al oleaje y al viento. En algunos lugares muy expuestos la zona supralitoral puede llegar hasta los 15-20 m de altura.
- Zona mediolitoral: entre 3,7 m y 0,9 m sobre el punto cero de bajamar. Prácticamente cada día queda cubierta durante la pleamar y al descubierto durante la marea baja.

- Franja infralitoral: entre 0,9 m y 0 m (punto cero de bajamar), y queda al descubierto durante la bajamar de mareas de intensidad media-alta. Corresponde a la parte más alta de la zona infralitoral, que se extiende por debajo de los $0 \mathrm{~m}$ hasta los $20 \mathrm{~m}$ de profundidad y siempre queda sumergida bajo el mar.

Los seres vivos que habitan el ecosistema intermareal, a caballo entre los ecosistemas terrestres y acuáticos, han de estar adaptados para vivir en condiciones del medio muy cambiantes. Así, si mientras están sumergidos están sometidos a condiciones de temperatura, salinidad, contenido en oxígeno, humedad... relativamente estables, que pueden cambiar progresivamente a lo largo del año, durante la bajamar han de hacer frente a la desecación y están expuestos a condiciones atmosféricas que pueden cambiar rápidamente. Es por ello que la di- 
versidad en las zonas altas del intermareal es muy baja y va aumentando a medida que el tiempo de inmersión se incrementa (Tabla 3).

Muy pocas especies viven en la zona supralitoral. Está colonizada casi exclusivamente por líquenes que forman bandas de colores distintos según domine una especie u otra. En la parte más alta Xanthoria parietina (L.) Beltr., 1858 forma un cinturón de color naranja/amarillo; la zona media está dominada por Caloplaca spp. y Lecanora spp., formando un cinturón de color naranjagrisáceo, donde se distinguen algunas manchas negras de Lichina confinis (O.F. Müller) C. Agardh, 1821; finalmente, en la parte más baja, hay un cinturón de color negro formado por Verrucaria maura Wahlenberg, 1803 que se adentra en la franja supralitoral (Gorostiaga \& Renobales, 1987; Renobales \& Noya, 1993).

En la parte más baja de esta zona (la más cercana a la franja supralitoral) pueden encontrarse algunos individuos del pequeño gasterópodo Melarhaphe neritoides (Linnaeus, 1758) y de la lapa Patella rustica Linnaeus, 1758, más abundantes en la franja supralitoral. La anchura del cinturón de $V$. maura es indicativa del grado de exposición al oleaje, y en lugares muy expuestos y abruptos esta zona puede extenderse bastantes metros sobre el nivel del mar [en los acantilados de Jaizkibel se ha encontrado a más de $12 \mathrm{~m}$ sobre el nivel cero de marea (AZTI-SIO-INSUB-UPV/EHU, 1993)]

La franja supralitoral queda ya bajo la influencia de las mareas, aunque solamente queda sumergida bajo el mar durante las pleamares de mareas vivas. Todavía son pocas las especies capaces de sobrevivir y desarrollarse en esta zona, aunque son más numerosas que en la zona anterior. El liquen $V$. maura y el molusco gasterópodo $M$. neritoides son especies características de esta franja, y, junto a ellas, son abundantes también el pequeño molusco bivalvo Lasaea adansoni (Gmelin, 1791) y la lapa Patella vulgata Linnaeus, 1758. Algunas especies de algas como la clorofita Blidingia minima (Nägeli ex Kützing) Kylin, 1947 y la rodofita Porphyra spp. forman notables cinturones estacionales.

Debajo de la franja supralitoral se extiende la zona mediolitoral, que corresponde a la zona intermareal en sentido estricto y está estrechamente unida al ritmo de las mareas: es cubierta por el mar durante la pleamar y queda en seco durante la marea baja. Las condiciones ambientales son mucho menos rigurosas que las de la franja supralitoral, y, en consecuencia, los valores de los distintos parámetros estructurales de la comunidad, especialmente los relativos a la diversidad y riqueza específica, son más altos (Tabla 3).

En los niveles más altos de esta zona (mediolitoral superior) el pequeño cirrípedo Chthamalus spp. [C. stellatus (Poli, 1791) y C. montagui Southward, 1976] forma un denso y extenso cinturón, donde habita una densa población del pequeño gasterópodo $M$. neritoides, lo que da nombre a la comunidad (comunidad de Chthamalus-Melarhaphe); las zonas más altas están colonizadas por el liquen Lichina pygmaea (O.F.Müller) C.Agardh, 1820. Para hacer frente a la desecación $M$. neritoides vive en las grietas y dentro de los caparazones vacíos de Chthamalus, y puede llegar a alcanzar densidades de hasta 100.000 individuos $/ \mathrm{m}^{2}$. Un amplio cinturón de Chthamalus es indicativo de un alto grado de exposición. Otras especies comunes en la zona mediolitoral superior son las lapas, $P$. vulgata y Patella depressa Pennant, 1777, los bivalvos $L$. adansoni, Mytilus galloprovincialis Lamarck, 1819 (mejiIlón) y Mytilaster minimus (Poli, 1795), y los crustáceos anfípodos del género Hyale.

Aunque no es muy común en la costa vasca, en las paredes subverticales expuestas de Jaizkibel es fácil encontrar manchas más o menos desarrolladas del alga parda Fucus spiralis var. limitaneus (Montagne) I.M.PérezRuzafa, 2001. Esta pequeña fucácea puede formar un cinturón, más o menos conspícuo, acompañada de otras algas como el alga calcárea Lithophyllum byssoides (Lamarck) Foslie, 1900 y la cespitosa Caulacanthus ustulatus (Mertens ex Turner) Kützing, 1843.

El mediolitoral medio está caracterizado por un denso cinturón de Chthamalus, y su fauna asociada, donde pueden encontrarse manchas de algas rojas como L. byssoides y $C$. ustulatus. Asimismo, el alga parda incrustante Ralfsia verrucosa (Areschoug) Areschoug, 1845 forma manchas de color oscuro, mientras que el alga roja $\mathrm{Ne}$ malion helminthoides (Velley) Batters, 1902 se desarrolla en verano formando un conspícuo cinturón. Algunas de las especies animales características del mediolitoral superior, como los moluscos mitílidos M.galloprovincialis y M. minimus, y los crustáceos anfípodos del género Hyale, también son abundantes en esta zona. Además, entre las

\begin{tabular}{|c|c|c|c|c|c|c|c|}
\hline A & $\begin{array}{l}\text { Supralitoral } \\
\text { Chthamalus- } \\
\text { M. neritoides }\end{array}$ & $\begin{array}{l}\text { Mediolit. sup. } \\
\text { Chthamalus- } \\
\text { L. byssoides }\end{array}$ & $\begin{array}{c}\text { Mediolitoral medio } \\
\text { Corallina (ahora } \\
\text { Ellisolandia) elongata }\end{array}$ & $\begin{array}{c}\text { Mediolitoral infer. } \\
\text { Corallina (ahora } \\
\text { Ellisolandia) elongata }\end{array}$ & $\begin{array}{c}\text { Infralitoral } \\
\text { Gelidium corneum }\end{array}$ & $\begin{array}{l}\text { Infralitoral } \\
\text { Laminaria- } \\
\text { Cystoseira }\end{array}$ & $\begin{array}{c}\text { Circalitoral } \\
\text { Halopteris } \\
\text { filicina }\end{array}$ \\
\hline Densidad (ind. $\mathrm{m}^{2}$ ) & $2,000-60,000$ & $25,000-40,000$ & $35,000-55,000$ & $20,000-60,000$ & $300-5,000$ & $300-1,000$ & $100-3,000$ \\
\hline Riqueza especifica $\mathrm{n}^{\circ} \mathrm{spp}$. & $3-12$ & $15-30$ & $35-45$ & $50-65$ & $20-50$ & $25-50$ & $10-30$ \\
\hline Diversidad (Hd) & $1-1.5$ & $1.5-2.5$ & $2-3$ & $2.5-3.5$ & $1.5-4$ & $3-3.8$ & $1.5-3$ \\
\hline B & $\begin{array}{l}\text { Mediolit. sup. } \\
\text { Fucus spiralis } \\
\text { var. limitaneus }\end{array}$ & $\begin{array}{l}\text { Infralitoral } \\
\text { Cystoseira- } \\
\text { Halopteris }\end{array}$ & $\begin{array}{c}\text { Circalitoral } \\
\text { Halopteris filicina }\end{array}$ & $\begin{array}{c}\text { Circalitoral } \\
\text { Sabellaria spinulosa }\end{array}$ & & & \\
\hline Densidad (ind. $\mathrm{m}^{2}$ ) & $286-386$ & $836-1830$ & $138-842$ & 654 & & & \\
\hline Riqueza especifica $\mathrm{n}^{\circ} \mathrm{spp}$. & $13-20$ & $49-55$ & $10-32$ & 16 & & & \\
\hline Diversidad (Hd) & $2.8-2.9$ & $2.27-3.17$ & 2.27 & 2.43 & & & \\
\hline
\end{tabular}

Tabla 3. - Parámetros estructurales de las comunidades de fondo duro. A) Borja et al., (2004). B) AZTI-SIO-INSUB-UPV/EHU (1993),

3. Taula. Hondo gogorretako komunitate bentikoen egiturazko parametroak. A) Borja et al., (2004). B) AZTI-SIO-INSUB-UPV/EHU (1993). 
anfractuosidades de $L$. byssoides es abundante el poliqueto Syllis amica Quatrefages, 1866.

La parte baja de la zona mediolitoral (mediolitoral inferior) está dominada por la comunidad de Corallina (ahora Ellisolandia), que forma un denso y ancho cinturón de color rosáceo que se extiende hasta la franja infralitoral a todo lo largo del litoral de Jaizkibel. Esta comunidad, descrita por Crisp y Mwaiseje (1989), está caracterizada por las algas rojas calcáreas Ellisolandia elongata (J.Ellis \& Solander) K.R.Hind \& G.W.Saunders, 2013 y Lithophyllum incrustans R.A.Philippi, 1837, esta última incrustada a la roca. La comunidad faunística que vive en esta zona presenta valores de diversidad y riqueza específicos mucho más elevados que los registrados en los niveles superiores del intermareal, y normalmente son los más elevados del intermareal: hasta 70 especies distintas de la macrofauna han sido identificadas en muestras pertenecientes a esta comunidad (Tabla 3). Entre las especies que habitan en este horizonte se encuentran varias especies de lapas ( $P$. vulgata, P. depressa, P. aspera), el poliplacóforo Acantochitona fascicularis (Linnaeus, 1767), bivalvos como M. galloprovincialis, M. minimus, Musculus costulatus (Risso, 1826), Modiolula phaseolina (Philippi, 1844); el erizo de mar Paracentrotus lividus (Lamarck, 1816); el cnidario Actinia equina (Linnaeus, 1758) (en grietas y pozas); numerosas especies de poliquetos [Polyophthalmus pictus (Dujardin, 1839), Eulalia viridis (Linnaeus, 1767), Platynereis dumerilii (Audouin \& Milne Edwards, 1834), Lysidice ninetta Audouin \& Milne-Edwards, 1833 ...], de crustáceos anfípodos [Hyale perieri (Lucas, 1849), H. stebbingi Chevreux, 1888, Jassa falcata (Montagu, 1808), Caprella spp....], isópodos como Dynamene bidentata (Adams, 1800) y tanaidáceos como Tanais dulongii (Audouin, 1826)].

Los fondos de las pozas situadas en la zona mediolitoral están recubiertos por esta comunidad. Incrustada a las rocas y piedras se encuentra $L$. incrustans, sobre la que crece E. elongata. En las grietas y recovecos encuentra refugio el erizo $P$. lividus, que cuando la roca es más blanda forma cavidades redondeadas, rascándola con sus dientes, en las que se guarece. Muchas veces se colocan sobre sí mismos pequeñas piedras y conchas de lapas como elementos de protección y camuflaje.

La parte más baja de la zona intermareal corresponde a la franja infralitoral, que solamente queda al descubierto durante la bajamar de las mareas vivas. En su parte superior las especies de algas dominantes del mediolitoral medio (E. elongata y L. incrustans) todavía tienen una presencia significativa. Junto a éstas, otras especies de algas como Pterosiphonia complanata (Clemente) Falkenberg, 1897 y Cladophora spp. forman pequeñas manchas. Más abajo da comienzo el cinturón que forma el alga roja Gelidium corneum (Hudson) J.V.Lamouroux, 1813 (antes G. sesquipedale) que se extiende por toda la zona infralitoral. Asociado a este cinturón se encuentra el alga roja calcárea Mesophyllum lichenoides (J.Ellis) Me.Lemoine, 1928. Las matas de Gelidium muchas veces están epifitadas por el alga roja Plocamium cartilagineum (Linnaeus) P.S.Dixon, 1967 y por las colonias del briozoo Electra pilosa (Linnaeus, 1767), dándole un color blancuzco a la base de las matas de Gelidium. Los valores de diversidad y riqueza específica de esta comunidad también son altos, aunque normalmente algo más bajos que los de la comunidad de Ellisolandia (Tabla 3). Algunas de las especies de la fauna del mediolitoral también son abundantes, así el molusco M. minimus y los crustáceos Caprella spp., Hyale spp., J. falcata.... Esta comunidad de G. corneum se extiende a la zona submareal.

\section{Comunidades submareales}

Los fondos rocosos submareales más someros de la costa de Gipuzkoa, y también los de Jaizkibel, presentan una vegetación homogénea compuesta principalmente por extensas praderas de G. corneum (Borja, 1987, 1988; Gorostiaga et al., 1988; Díez et al., 2003), cuyo mayor desarrollo se alcanza sobre fondos rocosos, muy expuestos y con una tasa de sedimentación de arena baja o moderada. Este horizonte de Gelidium, que comienza en la parte inferior de la franja infralitoral, llega a extenderse hasta los 10-15 m de profundidad, e incluso, mezclada con Cystoseira baccata (S.G.Gmelin) P.C.Silva, 1952, puede llegar a alcanzar los $25 \mathrm{~m}$ de profundidad (Borja et al., 1995, 2000, AZTI-SIO-INSUB-UPV/EHU, 1993). Las distintas especies de algas que forman parte de esta comunidad ocupan el espacio a través de una compleja estratificación vertical, en la que puede distinguirse un piso compuesto por las algas incrustantes M. lichenoides y Zanardinia typus (Nardo) P.C.Silva, 2000, un segundo piso, poco desarrollado, formado por P. complanata, E. elongata, Palmaria palmata (Linnaeus) Weber \& Mohr, 1805 y Cryptopleura ramosa (Hudson) L.Newton, 1931, y finalmente el piso formado por Gelidium corneum, bien desarrollado y que en primavera y verano está pobremente epifitado por Dictyota dichotoma (Hudson) J.V.Lamouroux, 1809 y P. cartilagineum, respectivamente (Borja et al., 2004). Hasta más del $70 \%$ de la totalidad de la cobertura algal está formado por G. corneum y M. Iichenoides. La macrofauna constituyente de esta comunidad es muy diversa (Tabla 3) y está compuesta, entre otras especies, por los poliquetos Spirobranchus polytrema (Philippi, 1844), Serpula concharum Langerhans, 1880, L. ninetta y P. dumerilii; moluscos, como Bittium reticulatum (da Costa, 1778), M. costulatus y Rocellaria dubia (Pennant, 1777); esponjas como Clathrina coriacea (Montagu, 1818) y Halichondria panicea (Pallas, 1766); cnidarios como Corynactis viridis Allman, 1846; crustáceos como Apherusa jurinei Milne-Edwards, 1830 y Caprella fretensis Stebbing, 1878; varias especies de briozoos de los géneros Crisia y Scrupocellaria y equinodermos como Ophiotrix fragilis (Abildgaard, in O.F. Müller, 1789). Esta comunidad se encuentra actualmente en regresión, debido a factores climáticos (disminución de insolación y aumento del oleaje) y presiones humanas (explotación y vertidos), lo que hace que en los últimos años la biomasa en la zona de Jaizkibel sea la menor de la serie desde 1983 (Borja et al., 2013).

Desde el límite inferior de la comunidad de Gelidium hasta 25-40 m de profundidad, y en zonas expuestas 
como es la costa de Jaizkibel, los fondos rocosos están cubiertos por distintas comunidades bentónicas que forman una zonación continua de macroalgas y macrobentos que pueden definirse por la vegetación que la caracteriza: fondos caracterizados por la presencia de Cystoseira baccata, C. baccata-Laminaria ochroleuca Bachelot de la Pylaie, 1824 y Halopteris filicina (Grateloup) Kützing, 1843 (Borja et al., 2004). Estas comunidades han sido encontradas en la zona oriental de Jaizkibel (AZTI-SIO-INSUB-UPV/EHU, 1993). Así, en un transecto submareal realizado a unos $25 \mathrm{~m}$ de profundidad, al este de Punta Sugur, se observa una zonación continua de macroalgas y macrobentos, donde puede distinguirse una zona dominada por $G$. corneum en la parte más alta de la laja (20 m); en la rampa descendente que le sigue L. ochroleuca es la especie más destacada (1-2 ejemplares $/ \mathrm{m}^{2}$ ). En la parte superior de la rampa a esta alga le acompañan Punctaria latifolia Greville, 1830 y D. dichotoma, que al aumentar la profundidad dejan paso a $C$. baccata y Dictyopteris polypodioides (A.P.De Candolle) J.V.Lamouroux, 1809. A partir de la cota $22 \mathrm{~m}$ aparece $H$. filicina que va aumentando su densidad con la profundidad. Entre la megafauna asociada destacan los equinodermos Sphaerechinus granularis (Lamarck, 1816) y Echinaster sepositus (Retzius, 1783), y entre la macrofauna los moluscos $B$. reticulatum (346 individuos $/ \mathrm{m}^{2}$ ) y M. costulatus (88 individuos $/ \mathrm{m}^{2}$ ), el poliqueto Salmacina dysteri (Huxley, 1855) (98 individuos/m²), crustáceos de la familia Paguridae (89 individuos $/ \mathrm{m}^{2}$ ) y el anfípodo Elasmopus rapax Costa, 1853 (64 individuos/m²).

En otro transecto similar realizado más cerca del cabo Higuer, se pueden distinguir un horizonte dominado por $C$. baccata y otro más profundo caracterizado por $H$. filicina y $D$. polypodioides. En algunas zonas el fondo está cubierto por manchas de $\mathrm{H}$. filicina y rodofíceas de pequeño porte. Entre la macrofauna destacan los moluscos B. reticulatum (1237 individuos $/ \mathrm{m}^{2}$ ), Nassarius incrassatus (Strøm, 1768) (101 individuos $/ \mathrm{m}^{2}$ ) y M. costulatus (50 individuos $/ \mathrm{m}^{2}$ ), y crustáceos de la familia Paguridae (107 individuos $/ \mathrm{m}^{2}$ ).

Galparsoro et al., (2009) consideran que actualmente esta comunidad únicamente está caracterizada por Cystoseira baccata (comunidad de Cystoseira spp.), ya que como consecuencia del calentamiento global la especie Laminaria ochroleuca, con preferencia por aguas más frías, ha desaparecido de la costa vasca.

En los mismos puntos, aunque a mayor profundidad (alrededor de la isobata de $40 \mathrm{~m}$ ), se realizaron otros dos transectos similares. En el situado junto a la Punta Sugur, a $42 \mathrm{~m}$ de profundidad, el fondo es llano, rocoso y con algunas bañeras de arena. Hay un ligero acúmulo de material fino sobre el sustrato rocoso. Sobre los bloques y cantos pequeñas matas de $H$. filicina y plantas aisladas de rodofíceas como Phyllophora crispa (Hudson) P.S.Dixon, 1964, Callyblepharis, Rhodimenia...

La megafauna está constituida también por S. granularis y E. sepositus, además de la gorgonia Eunicella verrucosa (Pallas, 1766). En las bañeras arenosas, nu- merosos ejemplares del equinodermo Holothuria spp. Entre la macrofauna destacan los moluscos Modiolula phaseolina (Philippi, 1844) (400 individuos $/ \mathrm{m}^{2}$ ) y B. reticulatum (205 individuos $/ \mathrm{m}^{2}$ ), el poliqueto Sabellaria spinulosa Leuckart, 1849 (50 individuos $/ \mathrm{m}^{2}$ ), el sipuncúlido Aspidosiphon muelleri Diesing, 1851 (60 individuos $/ \mathrm{m}^{2}$ ), y crustáceos de la familia Paguridae (43 individuos $/ \mathrm{m}^{2}$ )

El sustrato del transecto situado más cerca del cabo Higuer, a 43 m de profundidad, también es llano, rocoso, con zonas de arena. Algunas matas de pequeñas rodofíceas, P. crispa, Rhodymenia pseudopalmata (J.V.Lamouroux) P.C.Silva, 1952, tapizan las rocas, junto a varias especies de cnidarios como Gymnangium montagui (Billard, 1912) (abundante), E. verrucosa y Leptogorgia spp., y al erizo S. granularis. Entre la macrofauna destacan el poliqueto $S$. spinulosa (69 individuos $/ \mathrm{m}^{2}$ ) y los moluscos $B$. reticulatum (34 individuos $/ \mathrm{m}^{2}$ ) y M. phaseolina (18 individuos $/ \mathrm{m}^{2}$ ).

Estos dos últimos transectos corresponderían al límite inferior de la comunidad de Halopteris filicina. Según Borja et al., (2004) la comunidad de Halopteris se desarrolla en profundidades entre 25 y $50 \mathrm{~m}$ de profundidad. Por lo común, $H$. filicina está acompañada por rodófitas de pequeño porte como $P$. crispa y Peyssonnelia rubra (Greville) J.Agardh, 1851, y la fauna está constituida por el cirrípedo Verruca stroemia (O.F. Müller, 1776), que forma una capa que cubre las rocas, el sipuncúlido $A$. muelleri, el molusco bivalvo Modiolus barbatus (Linnaeus, 1758) y el cnidario Sertularella ellisii (Deshayes \& Milne Edwards, 1836), entre otros (Borja et al., 1995, 2000).

En la zona de Hondarribia, el fondo marino en las proximidades de la isobata - $45 \mathrm{~m}$ es heterogéneo, mezclándose las zonas de roca y arena, lo que se refleja en las comunidades faunísticas encontradas, donde predominan especies asociadas a este tipo de fondos, como el poliqueto tubícola filtrador Sabellaria spinulosa (AZTISIO-INSUB-UPV/EHU, 1993). Lo que señalaría la presencia de otra comunidad circalitoral de fondo duro que marca la transición hacia los fondos blandos: la comunidad de Sabellaria spinulosa. S, spinulosa es un poliqueto que vive en el interior de un tubo con forma de cono truncado que construye con granos de arena. Estos tubos pueden formar una densa costra (arrecife de S. spinulosa) sobre fondos rocosos, rocas y cantos circalitorales de medios moderadamente expuestos y con corrientes de marea de intensidad moderada. La dificultad del muestreo a esas profundidades, bien con escafandra o mediante dragas (Van Veen...), hace que dispongamos de poca información sobre la presencia de esta comunidad en la zona de Jaizkibel. De todas formas los resultados de AZTI-SIO-INSUB-UPV/EHU, (1993) señalan la presencia de esta comunidad en la zona.

A medida que la profundidad aumenta la intensidad lumínica disminuye, por lo que la cubierta de macrófitas va desapareciendo. Los sustratos rocosos van siendo ocupados paulatinamente por comunidades con dominancia fisionómica animal ricas en suspensívoros y filtradores, principalmente esponjas y cnidarios coloniales, aunque en 
la isobata $40 \mathrm{~m}$ de Jaizkibel, todavía persisten numerosas rodofíceas incrustantes. Profundidades próximas a los 25$30 \mathrm{~m}$ conllevan la aparición de invertebrados de cierto porte ausentes en niveles más superficiales del litoral, como los antozoos Alcyonium glomeratum (Hassal, 1843), Eunicella gazella Studer, 1878, E. verrucosa y Paramuricea grayi (Johnson, 1861), o diversidad de hidrozoos [Aglaophenia tubulifera (Hincks, 1861), Halecium liouvillei Billard, 1934, Halopteris catharina (Johnston, 1833), Lafoea dumosa (Fleming, 1820)] (Aguirrezabalaga et al., 1986, 1987; Altuna Prados, 1993, 1994a).

Comunidades paralelas de dominancia animal ricas en animales coloniales pueden establecerse en enclaves esciáfilos en niveles superiores del litoral, incluida la zona intermareal. Estas comunidades son abundantes en el cabo de Higuer y zonas próximas en extraplomos, pequeñas grutas y abrigos. La variabilidad faunística de estos poblamientos es notable, y está condicionada por factores edáficos difícilmente cuantificables (luminosidad, hidrodinamismo, sedimentación) que dan lugar a una gran heterogeneidad fisionómica con diferentes facies ricas en hidrozoos (Aglaophenia spp., Eudendrium capillare Alder, 1856, Obelia dichotoma (Linnaeus, 1758), Plumularia setacea (Linnaeus, 1758), Sertularella spp.), coralimorfarios (Corynactis viridis Allman, 1846), madreporarios [Polycyathus muellerae (Abel, 1959)], zoantídeos [Epizoanthus spp., Parazoanthus axinellae (Schmidt, 1862)], cirrípedos [Perforatus perforatus (Bruguière, 1789)], briozoos [Bugula spp., Chartella spp., Turbicellepora magnicostata (Barroso, 1919)], esponjas [Clathrina coriacea (Montagu, 1818), Chondrosia sp., Dysidea spp., Hemimycale sp., Oscarella lobularis (Schmidt, 1862), Petrosia ficiformis (Poiret spp., 1789), Spongia spp., Verongia spp., etc.], poliquetos serpúlidos, etc. (Aguirrezabalaga et al., 1984, 1986, 1987; Altuna Prados, 1994a, 1994b). El porte arborescente de muchas de estas especies facilita la epibiosis, enriqueciéndose notablemente la comunidad.

\section{COMUNIDADES DE SUSTRATO BLANDO}

En el área de Jaizkibel, apenas hay superfícies intermareales cuyo sustrato esté constituido por sedimentos, ya sean arenas, más o menos gruesas o finas, o fangos. Solamente en la playa de Hondarribia y en la zona de
Azabaratza queda al descubierto durante la marea baja una superficie intermareal arenosa. En la parte baja de este intermareal arenoso, y extendiéndose hasta los 5-10 $\mathrm{m}$ de profundidad, en áreas muy expuestas, se desarrolla la comunidad de Pontocrates arenarius-Eurydice pulchra. Esta comunidad está caracterizada por la dominancia de especies de crustáceos como Pontocrates arenarius (Bate, 1858) y Eurydice pulchra Leach, 1815, que dan nombre a la comunidad, y otros crustáceos como Iphinoe spp., moluscos como Donax y poliquetos como Dispio uncinata Hartman, 1951 y Scolelepis mesnili (Bellan \& Lagardere, 1971).

Los factores ambientales del biotopo donde se desarrolla esta comunidad (hidrodinamismo alto, sustrato muy inestable) son muy estresantes, por lo que la abundancia, diversidad y riqueza específica de la comunidad son bajos (Tabla 4).

A medida que aumenta la profundidad el hidrodinamismo va perdiendo intensidad y, en consecuencia, el tamaño de las partículas que forman los sedimentos, en términos generales, va siendo menor. En su trabajo sobre las comunidades bentónicas de la plataforma continental de Gipuzkoa, Martínez et al. (2007) estudiaron las muestras de nueve estaciones situadas frente a la costa de Jaizkibel, entre 6 y 135 m de profundidad. Hasta los 15-20 m de profundidad, en lugares expuestos como la costa de Jaizkibel, el sedimento sigue estando formado por arenas mediasfinas limpias que están habitadas por una comunidad faunística relativamente empobrecida dominada por los crustáceos, entre los que podemos destacar al pagúrido Diogenes pugilator (Roux, 1829) (hasta $715 \mathrm{ind} / \mathrm{m}^{2}$ ), Euridice sp., Bathyporeia elegans Watkin, 1938, Hippomedon sp. y Urothoe brevicornis Bate, 1862, y los poliquetos Nephtys cirrosa (Ehlers, 1868), D. uncinata, Scolelepis bonnieri (Mesnil, 1896) y Polydora sp. Los moluscos son muy escasos. Los valores de los parámetros estructurales de la comunidad van siendo más altos (Tabla 4).

A mayor profundidad, en sedimentos más finos y estables, se desarrollan la comunidad lusitánica-boreal de Tellina tenuis y la comunidad de Venus fasciata. En la costa vasca, se hace difícil distinguir y separar estas dos comunidades (Martínez \& Adarraga, 2001; Borja et al., 2004) ya que son bastantes las especies que forman

\begin{tabular}{|c|c|c|c|c|c|}
\hline A & $\begin{array}{l}\text { Comunidad de } \\
\text { P. arenarius - } \\
\text { E. pulchra }\end{array}$ & $\begin{array}{l}\text { Comunidad de } \\
\text { Tellina - Venus }\end{array}$ & $\begin{array}{c}\text { Comunidad de } \\
\text { Amphiura }\end{array}$ & $\begin{array}{c}\text { Com. de A. crinita- } \\
\text { P. calliopae (ahora } \\
\text { P. bihanica) - D. arietina }\end{array}$ & \\
\hline Densidad (ind. $\mathrm{m}^{2}$ ) & $50-100$ & $150-500$ & $900-2,000$ & $500-1,000$ & \\
\hline Riqueza específica $\mathrm{n}^{\circ} \mathrm{spp}$. & $5-9$ & $22-42$ & $80-130$ & $75-120$ & \\
\hline Diversidad (Hd) & $1.5-2$ & $3-4$ & $5-5.7$ & $5.4-6$ & \\
\hline B & $\begin{array}{c}\text { Com. de arenas infralitorales } \\
\text { con Nepthys cirrosa - } \\
\text { Bathyporeia spp }\end{array}$ & $\begin{array}{l}\text { Comunidad de } \\
\text { Tellina - Venus }\end{array}$ & $\begin{array}{c}\text { Comunidad de } \\
\text { Amphiura }\end{array}$ & $\begin{array}{c}\text { Com. de A. crinita- } \\
\text { P. calliopae (ahora } \\
\text { P. bihanica) - D. arietina }\end{array}$ & $\begin{array}{c}\text { Comunidad de } \\
\text { arenas finas con } \\
\text { Amphioxus } \\
\end{array}$ \\
\hline Densidad (ind. $\mathrm{m}^{2}$ ) & 338 & 272 & 1389 & 631 & 548 \\
\hline Riqueza específica $\mathrm{n}^{\circ} \mathrm{spp}$. & 23 & 70 & 120 & 100 & 84 \\
\hline Diversidad (Hd) & 2.69 & 5.35 & 5,2 & 5.54 & 5.3 \\
\hline
\end{tabular}

Tabla 4. - Parámetros estructurales de las comunidades bentónicas de fondo blando. A) Borja et al., (2004). B) Martínez et al., (2007).

4. Taula. Hondo bigunetako komunitate bentikoen egiturazko parametroak. A) Borja et al., (2004). B) Martínez et al., (2007). 
parte de ambas (N. cirrosa, B. elegans, Echinocardium cordatum (Pennant, 1777), Processa parva Holthuis, 1951), y, además, las especies que identifican la comunidad están ausentes. En consecuencia, y siguiendo el criterio de Borja et al. (2004), consideraremos ambas comunidades dentro de la comunidad de Tellina-Venus, que se distribuye en los fondos sublitorales arenosos del sureste del Golfo de Bizkaia entre 10-70 m de profundidad. Las especies más características de esta comunidad en la costa vasca son los moluscos bivalvos Angulus fabula (Gmelin, 1791), Nucula sulcata Bronn, 1831, Spisula subtruncata (da Costa, 1778) y Mactra stultorum (Linnaeus, 1758); los poliquetos N. cirrosa, Nephtys hombergii Savigny in Lamarck, 1818, Magelona johnstoni Fiege, Licher \& Mackie, 2000, Magelona filiformis Wilson, 1959, Chaetozone gibber Woodham \& Chambers, 1994, Lumbrineris spp., Spiophanes bombyx (Claparède, 1870), Paradoneis armata Glémarec, 1966, Aricidea catherinae Laubier, 1967; los crustáceos Ampelisca brevicornis (Costa, 1853), Dyastilis spp., B. elegans; y los equinodermos $E$. cordatum y Amphipholis squamata (Delle Chiaje, 1828), entre otras. La estructura de la comunidad es más compleja, y los valores de la abundancia, riqueza específica y diversidad son más altos (Tabla 4).

Al aumentar la profundidad la proporción de finos del sedimento aumenta. Predominan las arenas fangosas y fangos, y en la costa vasca estos fondos están habitados por la comunidad de Amphiura y la comunidad de Auchenoplax crinita - Paradiopatra calliopae (ahora P. bihanica) - Ditrupa arietina.

En aguas de la costa vasca, y frente a Jaizkibel, la comunidad de Amphiura se distribuye entre 50-70 y 150 $\mathrm{m}$ de profundidad, en sedimentos muy finos. Martínez et al. (2007) señalan que en la costa vasca las especies del género Amphiura, que caracterizan a esta comunidad, son mucho menos abundantes $\left(<10\right.$ individuos $/ \mathrm{m}^{2}$ ) que en el norte de Europa, donde se describió esta biocenosis. Junto a estos equinodermos, la comunidad está formada por numerosas especies de poliquetos (Prionospio fallax Söderström, 1920, Prionospio ehlersi Fauvel, 1928, Galathowenia oculata (Zachs, 1923), Ampharete finmarchica (M. Sars, 1864), Levinsenia gracilis (Tauber, 1879), Paradoneis ilvana Castelli, 1985, Spiophanes kroyeri Grube, 1860, Chaetozone setosa Malmgren, 1867, Terebellides stroemii Sars, 1835 ...), de moluscos [Thyasira flexuosa (Montagu, 1803), Turritella communis Risso, $1826, . .$. ] y de crustáceos [Ampelisca tenuicornis Liljeborg, 1855, Callianassa subterranea (Montagu, 1808), Harpinia antennaria Meinert, 1890]. La importancia de los crustáceos en la comunidad se reduce, mientras que aumenta la de los poliquetos. Es una comunidad muy diversa (Tabla 4): hasta más de 100 especies han sido identificadas en las muestras de esta comunidad y los valores de la diversidad llegan a ser superiores a 5 bit/ind.

La comunidad de Auchenoplax crinita-Paradiopatra calliopae (ahora P. bihanica) -Ditrupa arietina representa la transición entre la plataforma continental y el talud continental. Es característica del sureste del Golfo de Bizkaia (Cornet et al., 1983) y se encuentra en fondos arenosos situados en el borde de la plataforma continental, entre 150-250 m de profundidad. En la costa vasca ha sido encontrada frente a Jaizkibel (Martínez \& Adarraga, 2001; Martínez et al., 2007). Al igual que la comunidad de Amphiura, es muy diversa, y los valores de los parámetros estructurales de la comunidad son muy altos (Tabla 4). Además de la tres especies que dan nombre a la comunidad, los poliquetos Pterolysippe vanelli (Fauvel, 1936) sensu Eliason, 1955, emend, Euchone incolor Hartman, 1965, Chone cf. infundibuliformis Krøyer, 1856, Sosane sulcata Malmgren, 1866, Euclymene spp. Litocorsa stremma Pearson, 1970, Nephtys kersivalensis McIntosh, 1908 y Paradiopatra hispanica (Amoureux, 1972), el sipuncúlido Onchnesoma steenstrupii steenstrupii Koren \& Danielssen, 1876, y el molusco Pulsellum lofotense (M. Sars, 1865) son característicos de esta comunidad; junto con bastantes de las especies que constituyen la comunidad de Amphiura, como los poliquetos G. oculata, A. finmarchica, T. stroemi, P, fallax, P. ehlersi, C. setosa, y el molusco T. flexuosa, entre otras.

Los valores de los parámetros estructurales de la comunidad son, también, muy altos (Tabla 4).

En zonas muy concretas de la plataforma continental, asociadas a los afloramientos rocosos, en zonas sometidas a corrientes de profundidad de cierta intensidad, el sedimento está constituido por granos de arena de tamaño medio-grueso. Este tipo de sedimentos se clasifican como arenas gruesas. La comunidad de arenas gruesas de Amphioxus que los habita está compuesta por numerosas especies, muchas de las cuales tienen vida intersticial. Entre éstas hay que destacar a numerosas especies de poliquetos de la familia Syllidae, familia que es muy abundante en los sustratos rocosos y mucho menos frecuente en fondos blandos. El cefalocordado Branchiostoma lanceolatum (Pallas, 1774) (Amphioxus) es la especie más característica de la comunidad, y la que le da nombre. Además, otras muchas especies encuentran su hábitat preferente en estas arenas, sobre todo poliquetos y crustáceos. Los moluscos son menos abundantes. Entre los poliquetos cabe destacar a Pisione remota (Southern, 1914), Polygordius appendiculatus Fraipont, 1887, Protodorvillea kefersteini (Mclntosh, 1869), Glycera lapidum Quatrefages, 1866, Goniadella gracilis (Verrill, 1873), Mediomastus sp., Spio martinensis Mesnil, 1896, entre los crustáceos Haplostylus normani (Sars G.O., 1877), Diastylis laevis Norman, 1869, Euridice spp. y Anapagurus hyndmanni (Thompson, 1844), y el molusco Goodallia triangularis (Montagu, 1803). La riqueza específica y la diversidad son muy altas (Tabla 4).

\section{CONSIDERACIONES GENERALES}

El análisis de los resultados obtenidos en los distintos trabajos sobre las comunidades bentónicas marinas que se han realizado en la zona de Jaizkibel (Sarasua et al., 1984; Pérez Celorrio et al., 1985; AZTI-SIO-INSUBUPV/EHU 1993; Martínez \& Adarraga 2001; Martínez et al., 2007; Galparsoro et al., 2009...) permite afirmar que 
en el área de Jaizkibel se desarrollan muchas de las comunidades que se han descrito para los fondos rocosos y blandos de la plataforma continental de la costa vasca (Borja et al., 2004). Lo que expresa y muestra el valor biológico de los fondos marinos de Jaizkibel, como señalan Pascual et al. (2011). Teniendo como base toda la información biológica disponible sobre zooplancton, macrobentos, macroalgas, peces demersales, aves y cetáceos, estos autores realizaron el Mapa de Valoración Biológica (VB) de la plataforma continental y estuarios de la costa vasca. El método VB utilizado por los autores se basó en el de Derous et al. (2007), que ha sido aplicado en diferentes lugares de la costa europea atlántica. Pascual et al. (2011) concluyen que mientras que la Valoración Biológica (VB) integrada para el conjunto de la plataforma continental y estuarios de la costa vasca es baja, algunas áreas concretas, como la zona de Jaizkibel y algunas de las zonas más profundas del cañón de Capbretón, tienen una valoración alta o muy alta.

\section{AGRADECIMIENTOS}

A Álvaro Altuna, Ángel Borja y Mikel Marquiegui por su disposición y ayuda para la realización de este trabajo.

\section{BIBLIOGRAFÍA}

Aguirrezabalaga, F., Altuna, A., Borja, A., Feliú, J., García Carrascosa, A. M., Romero, A., San Vicente, C., Torres, L. A., Uriz, M. J. Ibáñez, M. 1984. Contribución al conocimiento de la fauna marina de la Costa Vasca II. Lurralde 7: 83-133.

Aguirrezabalaga, F., Altuna, A., Arrarás, M. D., Miguel, I., Romero, A., Ruiz de Ocenda, M. J., San Vicente, C., Ibáñez, M. 1986. Contribución al conocimiento de la fauna marina de la Costa Vasca IV. Lurralde 9: 133-158.

Aguirrezabalaga, F., Altuna, A., Martínez de Murguía, A., Romero, A, Zaballa, K., Ibáñez, M. 1987. Contribución al conocimiento de la fauna marina de la Costa Vasca V. Lurralde 10: 109-128.

Altuna Prados, A. 1991. Nota sobre la presencia de Paramuricea grayi (Johnson, 1861) (Cnidaria, Anthozoa), en la costa vasca. Munibe, Cienc. Nat. 43: 85-90.

Altuna Prados, A. 1994a. Estudio faunístico, ecológico y biogeográfico de los cnidarios bentónicos de la costa vasca. Tesis doctoral inédita. Universidad de Navarra. Pamplona.

Altuna Prados, A. 1994b. El orden Scleractinia (Cnidaria, Anthoza) en la costa vasca; consideraciones generales y especies litorales. Kobie 22: 67-82.

AZTI-SIO-INSUB-UPV/EHU, 1993. Campaña estival de medición de variables biológicas en dos zonas de la costa de Gipuzkoa próximas a Zumaia y Hondarribia. Informe inédito. Fundación Leonardo Torres Quevedo. Santander.

Borja, A. 1987. Cartografía, evaluación de la biomasa y arribazones del alga Gelidium sesquipedale (Clem.) Born. et Thur. en la costa guipuzcoana (N España). Investig. Pesq. 51: 199-224.

Borja, A. 1988. Cartografía y evaluación de la biomasa del alga Gelidium sesquipedale (Clem.) Born. et Thur. 1876 en la costa vizcaína (N España). Invest. Pesq. 52: 85-107

Borja, A., Gorostiaga, J. M. 1990. Distribución geográfica de Saccorhiza polyschides (Light.) Batt. en la costa vasca. Su po- sible relación con la temperatura. Actas del 6o Simposio Ibérico de estudios del Bentos Marino: 1-8. Palma de Mallorca.

Borja, A., Valencia, V., García, L., Arresti, A. 1995. Las comunidades bentónicas intermareales y submareales de San Sebastián-Pasajes (Guipúzcoa, norte de España). Actas del IV Coloquio Internacional de Oceanografía del Golfo de Vizcaya: 165-181.

Borja, A., Valencia, V., Castro, R., Franco, J., Bald, J., Uriarte, A., Mendizabal, M., Aguirrezabalaga, F. 2000. Establecimiento de las bases técnicas de conocimiento del área de San juan de Gaztelugatxe con vistas a su posible declaración como reserva marina. (Informes Técnicos/Departamento de Agricultura y Pesca, Gobierno Vasco; 87).

Borja, A., Aguirrezabalaga, F., Martínez, J., Sola, J. C., GarcíaArberas, L., Gorostiaga, J. M. 2004. In: Benthic communities, biogeography and resources management. A. Borja, M. Collins (Ed.): 455-492. (Oceanography and Marine Environment of the Basque Country, Elsevier Oceanography Series; 70).

Borja A. Fontán, A. Muxika, I. 2013 . Interactions between climatic variables and human pressures upon a macroalgae population: Implications for management. Ocean and Coastal Manag. 76: 85-95

Casares, C. 1987. Estudio de la flora bentónica marina de la costa guipuzcoana. Tesis Doctoral. Universidad de Barcelona.

Crisp D.J., Mwaiseje, B. 1989. Diversity in intertidal communities with special reference to the Corallina officinalis community. Scientia Mar. 53(2-3): 365-372.

Derous, S., Agardy, T., Hillewaert, H., Hostens, K., Jamieson, G., Lieberknecht, L., Mees, J., Moulaert, I., Olenin, S., Paelinckx, D., Rabaut M., Rachor, E., Roff, J., Stienen, E.W.M., Van der Walt, J.T., Van Lancker, V., Verfaillie, E., Vincx, M. Weslawski, J.M., Degraer, S. 2007. A concept for biological valuation in the marine environment. Oceanologia 49(1): 99-128.

Díez, I., Santolaria, A., Secilla, A., Gorostiaga, J.M. 2000. Comunidades fitobentónicas submareales de la zona exterior de la Reserva de la Biosfera de Urdaibai. Consideraciones sobre su estado ecológico. En: Investigación aplicada a la reserva de la Biosfera de Urdaibai. Gobierno Vasco (Ed.): 151-157. Vitoria.

Díez, I., Santolaria, A., Gorostiaga, J.M. 2003. The relationships of environmental factors to the structure and distribution of subtidal seaweed vegetation of the western Basque coast ( $\mathrm{N}$. Spain). Estuar. Coast. Shelf Sci. 56: 1041-1054.

Fischer-Piette, E. 1935. Quelques remarques bionomiques sur la côte basque française et espagnole. Bull. Lab. Saint Servan 14: 1-14.

Fischer-Piette, E. 1938. Sur le caractère méridional du bios intercotidal du Golfe de Gascogne. Comptes Rendus Soc. Biogéogr. Paris 15: 61-65.

Fischer-Piette, E. 1955a. Répartition le long des côtes septentrionales de l'Espagne des principales espèces peuplant les rochers intercotidaux. Ann. Inst. Océanogr. 31: 37-124

Fischer-Piette, E. 1955b Sur le déplacements de frontières biogéographiques intercotidales, observables en Espagne: situation en 1954-1955. Comptes Rendus Soc. Biogéogr. Paris 242 $2782-2784$

Fischer-Piette, E. 1956. Sur le déplacements de frontières biogéographiques intercotidales, actuallement en cours en Espagne: situation en 1956. Comptes Rendus Soc. Biogéogr. Paris 241: 447-449.

Fontán, A., Mader, J., González, M., Uriarte, A., Gyssels, P., Collins, M.B. 2006. Marine hydrodinamics betweeen San Sebastián and Hondarribia (Guipúzcoa, northern Spain): field measurements and numerical modelling. Scientia Mar. 70(Extra 1): 51-63. 
Fraga, F. 1981. Upwelling off the Galician coast, northwest Spain. In: Coastal upwelling. F.A. Richards (Ed.): 176-182. American Geophysical Union. Washington, D.C.

Galparsoro, I., Rodríguez, G., Borja, A., Muxika, I. 2009. Elaboración de mapas de hábitats y caracterización de fondos marinos de la plataforma continental vasca. Informe inédito elaborado por AZTI-Tecnalia para la Dirección de Biodiversidad; Viceconsejería de Medio Ambiente; Departamento de Medio Ambiente, Planificación Territorial, Agricultura y Pesca del Gobierno Vasco.

Galparsoro, I., Borja, A., Legorburu, I., Hernández, C., Chust, G., Liria, P., Úriarte, A. 2010. Morphological characteristics of the Basque continental shelf (Bay of Biscay, northern Spain); their implications for Integrated Coastal Zone Management. Geomorphology 118: 314-329

Gorostiaga, J.M., Renobales, G. 1987. Vegetación litoral: algas y líquenes de la Costa Vasca. En: Itsasoa. E. Ayerbe (Ed.): 81115. Ed. Etor. San Sebastián.

Gorostiaga, J.M., Casares, C., Fernández, J.A., Pérez, B., Sarasua, A. 1988. Sobre la expansión de Sargassum muticum (Yendo) Fensholt en la costa atlántica europea. Lurralde 11: 437-443.

Ibáñez, M. 1978. Características biogeográficas del litoral de la costa vasca. Lurralde 1: 121-127.

Ibáñez, M. 1989. Implicaciones biogeográficas de la continentalización de la costa vasca. Lurralde 12: 71-101.

Iribar X., Ibáñez, M. 1979. Subdivisión de la zona intermareal de San Sebastián en función de los datos obtenidos con mareógrafo. Actas del I Simposio Ibérico de Estudios del Bentos Marino. San Sebastián: 521-524.

Lewis, J.R. 1964. The ecology of rocky shores. Hodder \& Stoughton Educ. London.

Martínez, J., Adarraga, I. 2001. Distribución batimétrica de comunidades macrobentónicas de sustrato blando en la plata- forma continental de Guipúzcoa (Golfo de Vizcaya). Bol. Inst Esp. Oceanogr. 17: 33-48.

Martínez, J., Adarraga, I., Ruiz J.M. 2001. Tipificación de poblaciones bentónicas de los fondos blandos de la plataforma continental de Guipúzcoa (sureste del Golfo de Vizcaya). Bol. Inst. Esp. Oceanogr. 23: 85-110.

Pascual, M., Borja, A., Eede, S. V., Deneudt, K., Vincx, M., Galparsoro, I., Legorburu, I. 2011. Marine biological valuation mapping of the Basque continental shelf (Bay of Biscay), within the context of marine spatial planning. Estuar. Coast. Shelf Sci. 95 186-198.

Pérez Celorrio, B., Uriarte, A., Ibáñez, M. 1985. Estructura y variaciones estacionales del macrofitobentos intermareal en Fuenterrabía (Guipúzcoa). Lurralde 8: 61-80.

Renobales, G., Noya, R. 1993. Zonation of lichens on a calcareous rocky shore. Nova Hedwig. 57(3-4): 489-502.

San Vicente, C., Romero, A., Aguirrezabalaga, F., López, J.C. lbáñez, M. 1992. Macrobentos de los sustratos blandos infralitorales de los medios expuestos en la Costa Vasca. Actas del V Simposio Ibérico de Estudios del Bentos Marino. Sta Cruz de Tenerife: 273-293.

Sarasua, A., Alkain, I., Pérez, B., Fernández, J.A., Uriarte, A., San Millán, M.D., Ascacibar, M, Ibáñez, M. 1984. Estudio estructural del macrofitobentos intermareal de Guipúzcoa. Actas do IV Simposio Ibérico de Estudos do Benthos Marinho. Lisboa: 585-589.

Sauvageau, C. 1897. Note préliminaire sur les algues marines du Golfe de Gascogne. J. Bot. 11: 166-307.

Servain, J. 1977. Rapport preliminaire sur l'étude des données climatologiques historiques du proche Atlantique. (Rapport/ Universite de Bretagne Occidentale; 5).

Torres, J.A., Viera, L.I. 1998. Oiartzun Haranaren Geologia (Geología del Valle de Oiartzun). Oiartzungo Udala. (Mugarri; 5). 